NulklearMedizin

Nucl.-Med. Vol. $27 \quad 1988$

\title{
INHALTSVERZEICHNIS - CONTENTS
}

SACHREGISTER SUBJECT INDEX

AUTORENREGISTER INDEX OF AUTHORS 


\section{Inhalt - Contents}

Managing Editors: U. Büll, G. Hör, G. Hoffmann, H. Schicha, C.Schü̈michen_H Vetter

Universiläts-

Bibliothek

Münctien

\section{Grußwort}

F. Wolf 1

\section{Editorial}

U. Büll 2

3. Jahrestagung der Deutschen Gesellschaft für Nuklearmedizin

\section{Abstracts 7-54}

\section{Originalarbeiten - Original Articles}

S. Jinnouchi, H. Hoshi, K. Watanabe, T. Ueda, T. Yamaguchi: The Distribution of Radioiodine-Labeled N-Isopropyl-pIodoamphetamine in Permanently Ischemic Brain of the Mongolian Gerbil

Die Verteilung von Radiojod-markiertem $\mathrm{N}$-Isopropyl-p-Jodamphetamin im dauernd ischämischen Gehirn der mongolischen Wüstenmaus 5

C. Schuemichen, Th. Krause, G. Umbach, T. Wolff: Localization of ${ }^{99 \mathrm{~m}} \mathrm{Tc}$-Diphosphonates in Newly Formed Bone Matrix as a Measure of Bone Lesion Detectibiliy

Lokalisierung von ${ }^{99 \mathrm{~m}} \mathrm{Tc}$-Diphosphonaten in der frisch gebildeten Knochenmatrix als Maß für die Nachweiswahrscheinlichkeit von Knochenläsionen 8

G. Buttermann, J. Eiber, J. Hennig, H. W. Pabst: Doppelphotonen-Absorptiometrie: Eine neue Methode zur Bestimmung des Knochenmineralgehaltes. - I. Grundlagen

Dual-Photon Absorptiometry: A New Method of Determining Bone Mineral Content. - I. Fundamentals 12

G. Wahl. H. J. Biersack, B. Kozak, R. Paillard, H. Stich: Korrelieren szintigraphisch ermittelte Knochenstoffwechselaktivitäten mit der Kalzium- und Phosphorbestimmung durch die Röntgen-Mikroanalyse?

Is there any Correlation between Scintigraphically Observed Bone Metabolism and the Calcium and Phosphorus Content as Determined by X-Ray MicroAnalysis? 19

K. Mann, B. Saller, U. Mehl, R. Hörmann, E. Moser: Highly Sensitive De- termination of TSH in the Follow-Up of TSH-Suppressive Therapy of Patients with Differentiated Thyroid Cancer

Sensitive Bestimmung von TSH in der Überwachung einer TSH-suppressiven Hormontherapie bei Patienten mit differenziertem Schilddrüsenkarzinom 24

E. Kleinhans, M. Griese, W. Meyers, G. Weiller, U. Büll: Die Aussagekraft von basalem TSH und freiem $\mathrm{T}_{3}$ im Vergleich zur erweiteren In-vitro-Diagnostik bei der Erkennung von Schilddrüsenfunktionsstörungen

Value of Basal TSH and Free $\mathrm{T}_{3}$ in Detecting Thyroid Disorders as compared to Additional $\mathrm{T}_{4}$ Parameters 29

P. Tietze, K. F. Gratz, J. Freise, H. Hundeshagen: Ulkussuche mit ${ }^{99 \mathrm{~m}} \mathrm{Tc}$ Sucralfat

Ulcer Detection with ${ }^{99 \mathrm{~m}} \mathrm{Tc}$-Sucralfate 32

S. F. Akber: The Conundrum of Relaxation Times

Das Rätsel der Relaxationszeiten

U. Horstmeier, R. Klapdor, M. Bahlo, R. Montz: Immunoscintigraphy with Combinations of Various Monoclonal Antibodies - Studies on Xenografts of $\mathrm{Hu}$ man Gastrointestinal Carcinomas

Immunszintigraphie mit Mischungen verschiedener monoklonaler Antikörper Untersuchungen an Transplantaten menschlicher gastrointestinaler Karzinome 46

U. Buell, H. Braun, A. Ferbert, H. Stirner, C. Weiller, E. B. Ringelstein: Combined SPECT Imaging of Regional Cerebral Blood Flow $\left({ }^{99 m} \mathrm{Tc}-\mathrm{Hexame}\right.$ thyl-Prpyleneamine Oxime, HMPAO) and Blood Volume $\left({ }^{99 m} \mathrm{Tc}-\right.$ HexamethylPrpyleneamine Oxime, HMPAO) and Blood Volume ( $\left.{ }^{99 \mathrm{~m}} \mathrm{Tc}-\mathrm{RBC}\right)$ to Assess Regional Cerebral Perfusion Reserve in Patients with Cerebrovascular Disease

Kombinierte SPECT-Darstellung von regionaler Hirndurchblutung $\left({ }^{99 \mathrm{~m}} \mathrm{Tc}\right.$ HMPAO) und Blutvolumen $\left({ }^{99 \mathrm{~m}} \mathrm{Tc}\right.$ Erythrozyten) zur Abschätzung der zerebralen Perfusionsreserve bei Patienten mit zerebrovaskulären Erkrankungen 51
H. Klepzig jr., R. Standke, R. P. Baum, S. Tezak, D. Mildenberger, F. D. Maul, G. Hör, M. Kaltenbach: Vergleich von Belastungs-EKG und RadionuklidVentrikulographie bezüglich des Nachweises einer Myokardischämie bei isolierten Stenosen des Ramus interventricularis anterior

Comparison of Exercise ECG and Radionuclide Ventriculography in the Assessment of Myocardial Ischemia in $\mathrm{Pa}$ tients with Isolated Stenoses of the Left Anterior Descending Artery 57

C. L. Maini, G. Valle, C. Stella, R. Iovine, L. Marchetti: A Statistical Study on Left Ventricular Peak Filling Rate in Diabetes

Eine statistische Untersuchung über die linksventrikuläre »Peak Filling Rate« bei Diabetes 67

W. H. Knapp, K. Vyska, H.-J. Machulla, G. Notohamiprodjo, U. Schmidt, E.-J. Knust, U. Gleichmann: DoubleNuclide Study of the Myocardium Using ${ }^{201} \mathrm{Tl}$ and ${ }^{123}$ I-Labeled Fatty Acids in Non-Ischemic Myocardial Diseases

Untersuchung des Myokardes mit ${ }^{201} \mathrm{Tl}$ und ${ }^{123} \mathrm{~J}$-markierten Fettsäuren bei nichtischämischen Herzerkrankungen 72

P. B. Frederiksen, J. B. Christensen, H. Vejlsted, J. W. Rasmussen, B. Pedersen, C.-J. Edeling: ${ }^{57}$ Co-Bleomycin Scintigraphy for the Preoperative Detection and Staging of Lung Tumors

${ }^{57}$ Co-Bleomycin-Szintigraphie bei präoperativer Lungentumorsuche und -Klassifizierung 79

A. Hotze, B. Briele, F. Wolf, H. J. Biersack, F. F. Knapp: Localization and Activity of Inflammatory Bowel Disease Using ${ }^{111}$ In Leukocyte Imaging Lokalisation und Aktivität entzündlicher Darmerkrankungen mittels ${ }^{111}$ In-Leukozytenszintigraphie 83

M. Beyers, S. Aspinall, J. D. Conradie, P. J. Fourie: Radioiodination of Murine Anti-Alphafoetoprotein E.9 Monoclonal Antibody and Its $F\left(a b^{\prime}\right)_{2}$ Fragment for the Diagnosis of Hepatocellular Carcinoma 
Radiojodierung eines monoklonalen Antikörpers gegen ein Anti-Alphafetoprotein der Maus und seines $F\left(a^{\prime}\right)_{2}$-Fragments zur Diagnostik des hepatozellulären Karzinoms 87

E. Busse, R. P. Baum, G. Hör, B. Kornhuber: Nachweis der Bindung von ${ }^{131} \mathrm{~J}-$ Meta-Jod-Benzylguanidin an Strukturen der Plasmamembran von Neuroblastom-Zellinien

Demonstration of the Binding of ${ }^{131} \mathrm{I}$ Meta-Iodobenzylguanidine to Structures on the Plasma Membrane of Neuroblastoma Cell Lines 95

E. Moser, C. R. Pickardt, K. Mann, D. Engelhardt, C. M. Kirsch, P. Knesewitsch, K. Tatsch, T. Kreisig, Chr. Kurz, B. Saller: Ergebnisse der Radiojod-Behandlung von Patienten mit immunogener und nichtimmunogener $\mathrm{Hy}$ perthyreose bei Anwendung unterschiedlicher Herddosen

Results of Radioiodine Therapy of $\mathrm{Pa}$ tients with Immunogenic and Non-Immunogenic Hyperthyroidism Using Different Radiation Doses 98

H. Winker, P. Reuland, J. Müller, S. Weller, U. Feine: Die ${ }^{99 \mathrm{~m}}$ Tc-HMPAO Leukozytenszintigraphie in der Entzündungsdiagnostik am Skelettsystem - Erste Erfahrungen

Leukocyte Scintigraphy with ${ }^{99 \mathrm{~m}} \mathrm{Tc}$ HMPAO in the Diagnosis of Bone Inflammations - Early Results 121

G. Leinsinger, P. Schmiedek, T. Kreisig, K. Einhäupl, W. Bauer, E. Moser: ${ }^{133}$ Xe-DSPECT: Bedeutung der zerebrovaskulären Reservekapazität für Diagnostik und Therapie der chronischen zerebralen Ischämie

${ }^{133} \mathrm{Xe}$-DSPECT: Significance of the Cerebrovascular Reserve Capacity for the Diagnosis and Treatment of Chronik Cerebral Ischemia 127

T. M. D. Gimlette, M. Critchley, C. R. Aquire: Factors Predicting Hypothyroidism in Long-Term Follow-Up after ${ }^{131}$ I Therapy

Faktoren zur Voraussage der Hypothyreose in der Langzeitbeobachtung nach ${ }^{131} \mathrm{~J}$-Therapie 135

H. Luig, W. Eschner, M. Bähre, E. Voth, G. Nolte: Eine iterative Strategie zur Bestimmung der Quellverteilung bei der Einzelphotonen-Tomographie mit einer rotierenden Gammakamera (SPECT)

An Iterative Strategy for Determination of the Source Distribution in Single-Pho- ton Emission Tomography with a Rotating Gamma Camera (SPECT) 140

B. Palagi, F. Villa, P Verga, A. Broggi, F. Guzzinl, "C: Cozzi, A. Tomasi, R. Picozzi: Measurement of Glomerular Filtration. Rate by Impulse Synthesis: Clinical Validation ánd Optimization

Messung der glomerulären Filtrationsrate durch Impulssynthese: Klinische Validisierung und Optimierung 147

L. J. Anghileri, P. Thouvenot, F. Brunotte, J. Robert: On the Role of Transferrin in ${ }^{67} \mathrm{Ga}$ Uptake by Tumor Cells

Zur Rolle von Transferrin bei der ${ }^{67} \mathrm{Ga}$ Aufnahme durch Tumorzellen 151

Y. F. Shafiq, M. H. S. Al-Hissoni, M. H. Jassim: The Influence of Experimental Conditions on the Preparation of ${ }^{99 \mathrm{~m}} \mathrm{Tc}$ Pyrrolidino-Methyl Tetracycline [ ${ }^{99 \mathrm{~m}} \mathrm{Tc}$ PMT] in the Form of an Instant Kit

Der Einfluß experimenteller Bedingungen auf die Herstellung von ${ }^{99 m}$ Tc-Pyrrolidinmethyltetrazyklin [ $\left.{ }^{99 m} \mathrm{Tc}-\mathrm{PMT}\right] 154$

V. Kempi: Deconvolution Analysis of Renograms Obtained with Simultaneously Administered ${ }^{99 \mathrm{~m}}$ Tc-DTPA and ${ }^{131} \mathrm{I}$ Hippuran

Dekonvolutionsanalyse von Renogrammen nach gleichzeitiger Verabreichung von ${ }^{99 m}$ Tc-DTPA und ${ }^{131} \mathrm{~J}$-Hippuran 188

F. Palermo, F. Boccaletto, M. Magalini, G. Chiara, T. Tommaseo, L. Dapporto: Radioisotope Evidence of Varying Transit of Solid Food in Gastrectomized Patients with and without Dumping Syndrome

Radioisotoper Nachweis des variierenden Übergangs fester Nahrung bei Gastrektomie-Patienten mit und ohne Entleerungssyndrom 195

I. C. Dormehl, N. Hugo, A. van Gelder, M. Maree, R. de Winter, L. Jacobs: Scintigraphic Phase Analysis of Abnormal A-V Conduction in a Baboon

Szintigraphische Phasenanalyse eines atrioventrikulären Blocks bei einem Pavian 200

C. M. B. Duwel, F. C. Visser, M. J. van Eenige, J. C. Roos, J. P. Roos: Pacing and the Non-Invasive Evaluation of Myocardial Fatty Acid Metabolism by Means of $17-{ }^{123}$ I-Heptadecanoic Acid Scintigraphy

Pacing und die nichtinvasive Bestimmung des myokardialen Fettsäurestoffwechsels mittels der Szintigraphie mit $17^{123} \mathrm{~J}$ Heptadekansäure 204
Z. Szabó, G. Torsello, C. Reifenrath, R. Porschen, H. Vosberg: Experimentelle Untersuchung der Leberperfusion mit nichtdiffundierenden Radiotracern: Differenzierung der arteriellen und portal-venösen Perfusionskomponente durch Dekonvolutionsanalyse von FirstPass-Zeitaktivitätskurven

Experimental Investigation of the Perfusion of the Liver with Non-Diffusible Tracers - Differentiation of the Arterial and Portal-Venous Components by Deconvolution Analysis of First-Pass Time-Activity Curves 209

K. Tatsch, P. Knesewitsch, C.-M. Kirsch, G. Küffer, G. Dörner, A. Matuschke, J. Bogner, F. D. Goebel, E. Moser: Stellenwert der ${ }^{67} \mathrm{Ga}$-Szintigraphie in der Primärdiagnostik und Verlaufsbeurteilung opportunistischer Pneumonien bei Patienten mit AIDS

Value of ${ }^{67} \mathrm{Ga}$ Scintigraphy in Primary Diagnosis and Follow-Up of Opportunistic Pneumonias in Patients with AIDS 219

Th. Chatzipanagiotou, J. Müller-Brand, E. W. Radü, J. Rem, R. Fridrich: Zerebrale Durchblutung in Ruhe und nach $\mathrm{CO}_{2}$-Stimulation bei Patienten mit einseitiger Karotisobstruktion

Cerebral Blood Flow at Rest and after $\mathrm{CO}_{2}$ Stimulation in Patients with Unilateral Carotis Obstruction 232

S. Bielack, J. Knop, G. Delling, K. Winkler: Szintigraphische Verlaufskontrolle von Osteosarkomen während neoadjuvanter Chemotherapie - Ergebnisse der kooperativen Osteosarkomstudie COSS der Gesellschaft für Pädiatrische Onkologie

Scintigraphic Follow-Up of Osteosarcomas during Neoadjuvant Chemotherapy - Results of the Co-Operative Osteosarcoma Study (COSS) of the Society of Pediatric Oncology 237

P. Reuland, W. Müller-Schauenburg, H. Kühn, K. D. Keller, U. Feine: $180^{\circ}$ versus $360^{\circ}$-Rotation bei der SPECT des Skeletts

$180^{\circ}$ versus $360^{\circ}$ Rotation in Skeletal SPECT 242

F. Grünwald, H. Durwen, P. Bülau, A. Bockisch, C. E. Elger, A. Rohde, K. Reichmann, B. Ammari, A. Hotze, H. Penin, H.-J. Biersack: HMPAOSPECT bei zerebralen Anfällen

HMPAO-SPECT in Cerebral Seizures 248 
W. Becker, T. Steck, P. Albert, W. Börner: Hysterosalpingoscintigraphy: A Simple and Accurate Method Evaluating Fallopian Tube Patency

Hysterosalpingoszintigraphie: Eine einfache und genaue Methode zur Prüfung der Tubendurchgängigkeit 252

H. W. Müller-Gärtner, R. Montz, R. Klapdor, M. Hirschmann, W. Rehpenning, J. Langkowski: Radioimmunbehandlung solitärer Lebermetastasen mittels intratumoraler Instillation ${ }^{131} \mathrm{~J}$ markierter monoklonaler Antikörper Erste Ergebnisse einer klinischen Studie

Radioimmunotherapy of Solitary Liver Metastases by Means of Intratumoral Instillation of ${ }^{131} \mathrm{I}$-Labelled Monoclonal Antibodies - Preliminary Results of a Clinical Trial 258

F. Grünwald, J. Ruhlmann, B. Ammari, R. Knopp, A. Hotze, H.-J. Biersack: Erfahrungen mit einem »High Dose«Therapiekonzept beim metastasierenden differenzierten Schilddrüsenkarzinom

Experience with a High-Dose Concept of Differentiated Metastatic Thyroid Cancer Therapy 266

\section{Kurzmitteilungen -}

\section{Short Communications}

A. Hotze, A. Bockisch, M. Rüther, H. J. Biersack: Vergleich von ${ }^{99 \mathrm{~m}} \mathrm{Tc}$ HMPAO-markierten Leukozyten und ${ }^{99 \mathrm{~m}}$ Tc-Nanololloid bei Osteomyelitiden

Comparison of ${ }^{99 \mathrm{~m}} \mathrm{Tc}-\mathrm{HMPAO}-$ Labelled Leukocytes and ${ }^{99 m} \mathrm{Tc}$-Nanocolloid in Osteomyelitis 63
A. Behar, G. Lagrue, F. Cohen-Boulakia, J. Baillet: Capillary Filtration in Idiopathic Cyclic Edema - Effects of Daflon $500 \mathrm{mg}$

Kapillarfiltrierung beim idiopathischen zyklischen Ödem - Wirkung von Daflon $500 \mathrm{mg} \quad 105$

F. Duška, L. Hadaš, J. Vižda, P. Kafka, E. Urbanová, V. Palička, Y. Mazurová, M. Kuba, V. Grossman: ${ }^{99 m}$ Tc-Aminohexylidendiphosphonate and ${ }^{99 \mathrm{~m}} \mathrm{Tc}$ Pyrophosphate in the Scintigraphic Diagnosis of Experimental Myodardial Infarction in Dogs

${ }^{99 \mathrm{~m}}$ Tc-Aminohexylidendiphosphonat und ${ }^{99 m}$ Tc-Pyrophosphat zur szintigraphischen Diagnose des experimentellen Herzinfarkts beim Hund 226

M. Madler, S. Fritsch, G. Fenzl, A. Schalhorn, E. Moser: Perfusionszintigraphie mit ${ }^{99 \mathrm{~m}}$ Tc-markierten Mikrosphären zur Funktionsbeurteilung implantierter Portsysteme vor intraarterieller Zytostase bei Lebermetastasen

Perfusion Scintigraphy with ${ }^{99 \mathrm{~m}} \mathrm{Tc}$-Labeled Microspheres to Monitor Implanted Port Systems Prior to Intraarterial Cytostasis of Liver Metastases 228

\section{Berichte - Reports}

H. J. Biersack, U. Buell, P. J. Ell: HMPAO - Fundamental and Clinical Research

HMPAO - Grundlagen- und klinische Forschung 108

O. Schober, H. Hundeshagen: Qualitätskontrolle in vivo

In-Vivo Quality Control 157

\section{Fallberichte - Case Reports}

W. Becker, C. Pabst, W. Börner: Toxic Megacolon Presenting in the Leukocyte Scan as Distended Transverse Bowel

Das Erscheinungsbild eines toxischen Megakolons als dilatiertes Colon transversum im Leukozytenszintigramm 36

Th. Krause, C. Schümichen, S. Hohenloser, W. Kasper, Th. Meinertz: Szintigraphie mit ${ }^{111}$ In-markiertem Antimyosin $\mathrm{F}(\mathrm{ab})_{2}$ monoklonalem Antikörper und ${ }^{99 \mathrm{~m}}$ Tc-Pyrophosphat bei Rhabdomyolyse

Scintigraphy with ${ }^{111}$ In-Labeled Antimyo$\sin \mathrm{F}(\mathrm{ab})_{2}$ Monoclonal Antibody and ${ }^{99 m} \mathrm{Tc}$-Pyrophosphate in Rhabdomyolysis 38

P. A. Dimopoulos, S. D. Yarmenitis, P. Vasilakos, G. M. Zavras, Th. Gorilas: False Aneurysm of Splanchnic Arteries - A Scintigraphic Pitfall

Falsches Aneurysma der Mesenterialarterien - Eine szintigraphische Fehlerquelle 272

\section{Buchbesprechungen - Book Reviews}

$40,118,274$

\section{Kleine Mitteilungen - \\ Announcements}

$42,66,120,185,231,276$ 


\section{Sachregister - Subject Index}

\section{Originalarbeiten}

AIDS, Pneumonien, ${ }^{67} \mathrm{Ga} 219$

Amphetamin, ${ }^{131} \mathrm{~J}$, ischämisches Hirn, Maus 5

Antimyosin, ${ }^{111}$ In, Rhabdomyolyse 38

Biokinetik, ${ }^{131} \mathrm{~J}$ Amphetamin, ischämisches Hirn, Maus 5

Bleomycin, ${ }^{57} \mathrm{Co}$, Lungentumoren 79

Berichte:

HMPAO, Grundlagen und klinische Forschung 108

Qualitätskontrolle in vivo 157

Schilddrüse 158

Hirn 160

Radionuklidventrikulographie 161

Herz, ${ }^{20 I} \mathrm{Tl},{ }^{99 m} \mathrm{Tc}$-MIBI 162

Skelett 163

Leukozyten 164

Monoklonale Antikörper 169

Lunge 173

NMR 177

Buchbesprechungen:

Atlas of Radionuclide Imaging 274

Biologische Wirkungen von inhalierten Radionukliden 119

Fractionation in Radiotherapy 118

Functional Studies Using NMR 40

Genetic Effects from Internally Deposited Radionuclides 274

Klinische Kernspintomographie 41

Klinische Nuklearmedizin 40

Labelled Compounds and Radiopharmaceuticals Applied in Nuclear Medicine 118

Nuclear Medicine Annual 198741

Radiation Protection in Occupational Health 118

The Year Book of Nuclear Medicine 1987274

Tschernobyl und die Folgen - Begutachtung von Strahlenschäden 274

Tumoren der Lunge 41

Wandel nuklearmedizinische Nierendiagnostik 40

${ }^{57} \mathrm{Co}$-Bleomycin, Lungentumoren 79

$\mathrm{CO}_{2}$-Stimulation, einseitige Karotisobstruktion, zerebrale Perfusionsreserve 232

COSS-Studie, Osteosarkomstudie, szintigraphische Verlaufskontrolle unter Chemotherapie 237
Dekonvolutionsanalyse, Leberperfusion, experimentell 209

Dekonvolutionsanalyse, Renogramm 188

Diphosphonate, ${ }^{99 \mathrm{~m}} \mathrm{Tc}$, Knochenmatrix, Knochenläsion, Nachweiswahrscheinlichkeit 8

Doppelphotonen-Absorptiometrie, Knochenmineral, Grundlagen 12

DSPECT, ${ }^{133} \mathrm{Xe}$, zerebrovaskuläre $\mathrm{Re}$ servekapazität 127

Dumping-Syndrom, Transitzeit fester Nahrung 195

\section{Editorial: PET versus SPECT 2}

Fallbericht, falsches Aneurysma der Mesenterialarterien, pitfall 272

Fallbericht, Rhabdomyolyse, ${ }^{111}$ In-Antimyosin, ${ }^{99 \mathrm{~m}} \mathrm{Tc}$-Pyrophosphat 38

Fallbericht, toxisches Megakolon, Leukozytenszintigraphie 36

Fettsäuren, ${ }^{123} \mathrm{~J}$, Metabolismus 204

Fettsäuren, ${ }^{123} \mathrm{~J}$, nichtischämische Herzerkrankungen 72

${ }^{67} \mathbf{G a}$, Pneumonien, AIDS 219

${ }^{67} \mathrm{Ga}$, Transferrin, Tumoraufnahme 151

Glomeruläre Filtrationsrate, Messung durch Impulssynthese 147

Herz, siehe Myokard

HMPAO, siehe ${ }^{99 \mathrm{~m}}$ Tc-HMPAO

Hyperthyreose, Radiojodtherapie, Herddosen, Ergebnisse 98

Hypothyreose, Radiojodtherapie, Langzeitbeobachtung 135

Hysterosalpingoszintigraphie, Tubendurchgängigkeit 252

Immunszintigraphie, Anti-Alphafoetoprotein 87

Immunszintigraphie, ${ }^{111}$ In-Antimyosin, Rhabdomyolyse 38

Immunszintigraphie, Mischung verschiedener Antikörper 43

Immuntherapie, solitäre Lebermetastasen 258

Impulssynthese, Messung der glomerulären Filtrationsrate 147

${ }^{111}$ In-Antimyosin, ${ }^{99 \mathrm{~m}}$ Tc-Pyrophosphat, Rhabdomyolyse, Fallbericht 38

${ }^{111}$ In-Oxin, Leukozyten, entzündliche Darmerkrankungen 83

Iterative Rekonstruktion, SPECT 140
${ }^{131} \mathbf{J}$-Amphetamin, ischämisches Hirn, Maus 5

${ }^{123} \mathrm{~J}$-Fettsäuren, nichtischämische Herzerkrankungen 72

${ }^{123} \mathrm{~J}$-Fettsäuren, Metabolismus 204

${ }^{131} \mathrm{~J}$-Hippuran, Renogramm, Dekonvolutionsanalyse 188

${ }^{131} \mathrm{~J}-\mathrm{MIBG}$, Bindung an Neuroblastomzellen 95

${ }^{131} \mathrm{~J}$-Therapie, High-dose-Konzept, metastasierendes Schilddrüsenkarzinom 266

${ }^{131} \mathrm{~J}$, Therapie, Hyperthyreose, Herddosen, Ergebnisse 98

${ }^{131} \mathrm{~J}$-Therapie, Hypothyreose, Langzeitbeobachtung 135

Kapillarfiltrierung, Ödem 105

KHK, Radionuklid-Ventrikulographie, Belastungs-EKG 57

Knochen, Entzündung, Leukozyten, ${ }^{99 m}$ Tc-HMPAO 121

Knochen, Läsion, Nachweiswahrscheinlichkeit, ${ }^{99 \mathrm{~m}} \mathrm{Tc}$-Diphosphonate 8

Knochen, Osteosarkom, COSS-Studie 237

Knochen, SPECT, $180^{\circ}$ - versus $360^{\circ}$-Rotation 242

Knochenmineral, Doppelphotonen-Absorptiometrie, Grundlagen 12

Knochenmineral, Korrelation mit Knochenstoffwechsel 19

Knochenstoffwechsel, Korrelation mit Knochenmineral 19

Lebermetastasen, Immuntherapie 258

Lebermetastasen, intraarterielle Zytostase, Perfusionsszintigraphie zur Kontrolle 228

Leberperfusion, First-Pass, Dekonvolutionsanalyse 209

Leukozytenszintigraphie, ${ }^{111} \mathrm{In}$, entzündliche Darmerkrankungen 83

Leukozytenszintigraphie, ${ }^{99 \mathrm{~m}} \mathrm{Tc}$-HMPAO, Knochen, Entzündung 121

Leukozytenszintigraphie, ${ }^{99 \mathrm{~m}} \mathrm{Tc}-\mathrm{HMPAO}$, ${ }^{99 \mathrm{~m}}$ Tc-Nanokolloid 63

Leukozytenszintigraphie, toxisches Megakolon, Fallbericht 36

Lunge, Pneumonien, AIDS, ${ }^{67} \mathrm{Ga} 219$

Lungentumoren, ${ }^{57} \mathrm{Co}$-Bleomycin 79

Magenentleerung, Dumping-Syndrom, Transitzeit 195

Megakolon, toxisch, Leukozytenszintigraphie, Fallbericht 36

MIBG, ${ }^{131} \mathrm{~J}$, Bindung an Neuroblastomzellen 95 
Monoklonale Antikörper, Mischung, Immunszintigraphie 43

Myokard, ${ }^{123} \mathrm{~J}$-Fettsäuren, Metabolismus 204

Myokard, LAD Stenose, Radionuklidventrikulographie 57

Myokard, ${ }^{201} \mathrm{Tl}$, ${ }^{123} \mathrm{~J}$-Fettsäuren, nichtischämische Erkrankungen 72

Myokardinfarkt, experimentell, ${ }^{99 \mathrm{~m}} \mathrm{Tc}-$ Aminohexylidendiphosphonat, ${ }^{99 \mathrm{~m}} \mathrm{Tc}$ Pyrophosphat 226

NMR, Relaxationszeiten 43

Ödem, Kapillarfiltrierung 105

Osteomyelitis, Leukozytenszintigraphie, ${ }^{99 \mathrm{~m}}$ Tc-Nanokolloid 63

Osteosarkom, COSS-Studie, Sammelstatistik 237

PET, versus SPECT, Editorial 2

Phasenanalyse, Radionuklidventrikulographie, AV Block 200

Pitfall; falsches Aneurysma der Mesenterialarterien, Fallbericht 272

Portsysteme, Lebermetastasen, intraarterielle Zytostase, Perfusionsszintigraphie 228

Qualitätskontrolle, in vivo, Bericht 157

Radiojodtherapie, High-dose-Konzept, metastasierendes differenziertes Schilddrüsenkarzinom 266

Radiojodtherapie, Hypothyreose, Langzeitbeobachtung 135

Radiojodtherapie, Hyperthyreose, Herddosen, Ergebnisse 98

Radionuklid-Ventrikulographie, Belastungs-EKG, LAD Stenose 57

Radionuklid-Ventrikulographie, PeakFilling-Rate bei Diabetes 67

Radionuklid-Ventrikulographie, Phasenanalyse bei AV Block 200

Relaxationszeiten, NMR 43

Renographie, Dekonvolutionsanalyse 188

Rhabdomyolyse, ${ }^{111}$ In-Antimyosin, ${ }^{99 \mathrm{~m}} \mathrm{Tc}$ Pyrophosphat, Fallbericht 38

Schilddrüse, Funktionsdiagnostik, TSH basal mit $\mathrm{T}_{3} 29$

Schilddrüsenkarzinom, metastasierend, High-dose-Therapiekonzept 266

Schilddrüsenkarzinom, Nachsorge, TSH sensitiv 24

Skelett, siehe Knochen

SPECT, iterative Datenrekonstruktion 140

SPECT, Knochen, $180^{\circ}$ - versus $360^{\circ}$-Rotation 242

SPECT, ${ }^{99 \mathrm{~m}}$ Tc-HMPAO, zerebrale Anfälle 248

SPECT, versus PET, Editorial 2

SPECT, zerebrale Perfusionsreserve 46
${ }^{{ }^{m} \text { m }}$ Tc-Aminohexylidendiphosphonat, Myokardinfarkt, experimentell 226

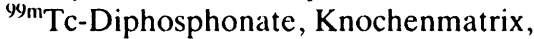
Knochenläsion, Nachweiswahrscheinlichkeit 8

${ }^{99 m}$ Tc-DTPA, Renogramm, Dekonvolutionsanalyse 188

${ }^{99 m}$ Tc-HMPAO, Grundlagen und klinische Forschung, Berichte 108

${ }^{99 \mathrm{~m}}$ Tc-HMPAO, Leukozyten, ${ }^{99 \mathrm{~m}}$ Tc-Nanokolloid 63

${ }^{99 \mathrm{~m}}$ Tc-HMPAO, Leukozyten, Knochen, Entzündung 121

${ }^{99 \mathrm{~m}}$ Tc-HMPAO, zerebrale Anfälle 248

${ }^{99 \mathrm{~m}}$ Tc-HMPAO, ${ }^{99 \mathrm{~m}}$ Tc-RBC, zerebrale Perfusionsreserve 46

${ }^{99 \mathrm{~m}}$ Tc-Mikrosphären, Lebermetastasen, intraarterielle Zytostase, Kontrolle 228

${ }^{99 \mathrm{~m}}$ Tc-PMT, Herstellung eines Instant Kits 151

${ }^{99 m}$ Tc-Pyrophosphat, ${ }^{111}$ In-Antimyosin, Rhabdomyolyse, Fallbericht 38

${ }^{99 \mathrm{~m}}$ Tc-Pyrophosphat, Myokardinfarkt, experimentell 226

${ }^{99}$ Tc-Sucralfat, Ulkussuche 32

TSH, basal, $\mathrm{T}_{3}$, Funktionsdiagnostik der Schilddrüse 29

TSH, sensitiv, Nachsorge, Schilddrüsenkarzinom 24

$\mathrm{T}_{3}$, TSH basal, Funktionsdiagnostik der Schilddrüse 29

Tubendurchgängigkeit 252

Tumor, ${ }^{67} \mathrm{Ga}$-Aufnahme, Transferrin 151

Ulkus, -suche, ${ }^{99 \mathrm{~m}} \mathrm{Tc}$-Sucralfat 32

${ }^{133} \mathbf{X e - D S P E C T , ~ z e r e b r o v a s k u l a ̈ r e ~} \mathrm{Re}$ servekapazität 127

Zerebrale Anfälle, ${ }^{99 \mathrm{~m}} \mathrm{Tc}$-HMPAO 248

Zerebrovaskuläre Erkrankungen, chronisch, ${ }^{133} \mathrm{Xe}$-DSPECT, Reservekapazität 127

Zerebrovaskuläre Erkrankungen, einseitige Karotisobstruktion, $\mathrm{CO}_{2}$-Stimulation, Perfusionsreserve 232

Zerebrovaskuläre Erkrankungen, zerebrale Perfusionsreserve 46

\section{Subject Index}

\section{Original Articles}

AIDS, pneumonia, ${ }^{67} \mathrm{Ga} 219$

Amphetamine, ${ }^{131} \mathrm{I}$, ischemic brain, mouse 5

Antimyosin, ${ }^{111}$ In, rhabdomyolysis 38

Biokinetics, ${ }^{131} \mathrm{I}$-amphetamine, ischemic brain, mouse 5

Bleomycin, ${ }^{57} \mathrm{Co}$, lung tumors 79

Bone, inflammation, leukocytes, ${ }^{99 \mathrm{~m}} \mathrm{Tc}$ HMPAO 121

Bone, lesion detectability, ${ }^{99 \mathrm{~m}} \mathrm{Tc}$-diphosphonates 8

Bone metabolism, correlation with bone mineral 19

Bone mineral, correlation with bone metabolism 19

Bone mineral, dual photon absorptiometry, fundamentals 12

Bone, osteosarcoma, COSS study 237

Bone, SPECT, $180^{\circ}$ versus $360^{\circ}$ rotation 242

Book Reviews: Atlas of Radionuclide Hepatobiliary Imaging 274

Biologische Wirkungen von inhalierten Radionukliden 119

Fractionation in Radiotherapy 118

Functional Studies Using NMR 40

Genetic Effects from Internally Deposited Radionuclides 274

Klinische Kernspintomographie 41

Klinische Nuklearmedizin 40

Labelled Compounds and Radiopharmaceuticals Applied in Nuclear Medicine 118

Nuclear Medicine Annual 198741

Radiation Protection on Occupational Health 118

The Year Book of Nuclear Medicine 1987274

Tschernobyl und die Folgen - Begutachtung von Strahlenschäden 274

Tumoren der Lunge 41

Wandel nuklearmedizinischer Nierendiagnostik 40

CAD, equilibrium radionuclide ventriculography, exercise ECG 57

Capillary filtration, edema 105

Case Report:

False aneurysma of splanchnic arteries, pitfall 272 
Toxic megacolon, leukocyte scintigraphy 36

Rhabdomyolysis, "11 In-antimyosin, ${ }^{99 m}$ Tc-pyrophosphate 38

Cerebral seizures, ${ }^{99 \mathrm{~m}} \mathrm{Tc}$-HMPAO 248

Cerebrovascular disease, cerebral perfusion reserve 46

Cerebrovascular disease, chronic, ${ }^{133} \mathrm{Xe}$ DSPECT, reserve capacity 127

Cerebrovascular disease, unilateral carotis obstruction, $\mathrm{CO}_{2}$-stimulation, perfusion reserve 232

${ }^{57} \mathrm{Co}$-bleomycin, lung tumors 79

$\mathrm{CO}_{2}$-stimulation, unilateral carotis obstruction, cerebral perfusion reserve 232

COSS study, osteosarcoma, scintigraphic follow-up during chemotherapy 237

Deconvolution analysis, perfusion of liver, experimental 209

Deconvolution analysis, renogram 188

Diphosphonates, ${ }^{99 \mathrm{~m}} \mathrm{Tc}$, bone matrix, bone lesion detectability 8

DSPECT, ${ }^{133} \mathrm{Xe}$, cerebrovascular reserve capacity 127

Dual-photon absorptiometry, bone mineral, fundamentals 12

Dumping syndrome, transit time of solid food 195

Edema, capillary filtration 105

Editorial: PET versus SPECT 2

Equilibrium radionuclide ventriculography, exercise, ECG, LAD stenosis 57

Equilibrium radionuclide ventriculography, phase analysis in abnormal A-V conduction 200

Equilibrium radionuclide ventriculography, peak filling rate in diabetes 67

Fatty acids, ${ }^{123} \mathrm{I}$, metabolism 204

Fatty acids, ${ }^{123}$ I, non-ischemic myocardial diseases 67

${ }^{67} \mathbf{G a}$, pneumonia, AIDS 219

${ }^{67} \mathrm{Ga}$, transferrin, tumor-uptake 151

Glomerular filtration rate, measurement by impulse synthesis 147

Heart, see myocardium

HMPAO, see ${ }^{99 \mathrm{~m}}$ Tc-HMPAO

Hyperthyroidism, radioiodine therapy, doses, results 98

Hysterosalpingoscintigraphy, tube patency 252

Hypothyroidism, radioiodine therapy, long-term follow-up 135

${ }^{131} \mathbf{I}$-amphetamine, ischemic brain, mouse

${ }^{123}$ I-fatty acids, metabolism 204

${ }^{123}$ I-fatty acids, non-ischemic myocardial diseases 67
${ }^{131}$ I-hippuran, renogram, deconvolution analysis 188

${ }^{131}$ I-therapy, high-dose concept, metastatic thyroid carcinoma 266

${ }^{131}$ I-therapy, hyperthyroidism, doses, results 98

${ }^{131}$ I-therapy, hypothyroidism, long-term follow-up 135

Immunodetection, see immunoscintigraphy

Immunoscintigraphy, anti-alphafoetoprotein 87

Immunoscintigraphy, combination of various antibodies 43

Immunoscintigraphy, ${ }^{111}$ In-antimyosin, rhabdomyolysis 38

Immunotherapy, solitary liver metastases 258

Impulse synthesis, measurement of glomerular filtration rate 147

${ }^{111}$ In-antimyosin, ${ }^{99 \mathrm{~m}} \mathrm{Tc}$-pyrophosphate, rhabdomyolysis, case report 38

${ }^{111}$ In-oxine, leukocytes, inflammatory bowel disease 83

Iterative reconstruction, SPECT 140

Leukocyte scintigraphy, ${ }^{111}$ In inflammatory bowel disease 83

Leukocyte scintigraphy, ${ }^{99 \mathrm{~m}} \mathrm{Tc}$-HMPAO, bone, inflammation 121

Leukocyte scintigraphy, ${ }^{99 \mathrm{~m}} \mathrm{Tc}$-HMPAO, ${ }^{99 m}$ Tc-nanocolloid 63

Leukocyte scintigraphy, toxic megacolon case report 36

Liver metastases, immunotherapy 258

Liver metastases, intraarterial cytostasis, perfusion scintigraphy for control 228

Liver perfusion, first-pass, deconvolution analysis 209

Lung, pneumonia, AIDS, ${ }^{67} \mathrm{Ga} 219$

Lung tumors, ${ }^{57} \mathrm{Co}$-bleomycin 79

Megacolon, toxic, leukocyte scintigraphy, case report 36

MIBG, ${ }^{131}$ I, binding to neuroblastoma cells 95

Monoclonal antibodies, combination, immunoscintigraphy 43

Myocardial infarction, experimental, ${ }^{99 \mathrm{~m}} \mathrm{Tc}$-aminohexylidendiphosphonate, ${ }^{99 m}$ Tc-pyrophosphate 226

Myocardium, ${ }^{123}$ I-fatty acids, metabolism 204

Myocardium, LAL stenosis, equilibrium radionuclide ventriculography 57

Myocardium, ${ }^{201} \mathrm{Tl},{ }^{123} \mathrm{I}$-fatty acids, nonischemic diseases 67

NMR, relaxation times 43

Osteomyelitis, leukocyte scintigraphy, ${ }^{99 \mathrm{~m}}$ Tc-nanocolloid 63

Osteosarcoma, COSS study, multicentric results 237
PET, versus SPECT, Editorial 2

Pitfall, false aneurysma of splanchnic arteries, case report 272

Port systems, liver metastases, intraarterial cytostasis, perfusion scintigraphy 228

Quality control, in vivo, reports 157

Radioiodine therapy, high-dose concept, differentiated metastatic thyroid carcinoma 266

Radioiodine therapy, hyperthyroidism, doses, results 98

Radioiodine therapy, hypothyroidism, long-term follow-up 135

Radionuclide ventriculography, see equilibrium-radionuclide-ventriculography

Relaxation times 43

Renography, deconvolution analysis 188

Reports:

In vivo quality control (only German, no English summary) 157

HMPAO - fundamental and clinical research 108

Pharmacological development 108

Kinetics baboon rat 109

Intracellular localization 110

Wada test 110

Brain death 111

Clinical data 113

Labelling of leukocytes:

Inflammatory bowel disease 113

Abscesses and lesions 115

Abdominal diseases 115

Trapping mechanism 116

Tumor detection, blood flow 116

Liver tumors 117

Rhabdomyolysis, ${ }^{111}$ In-antimyosin, ${ }^{99 \mathrm{~m}} \mathrm{Tc}$ pyrophosphate, case report 38

Skeleton, see bone

SPECT, bone, $180^{\circ}$ versus $360^{\circ}$ rotation 242

SPECT, cerebral perfusion reserve 46

SPECT, iterative data reconstruction 140

SPECT, ${ }^{99 \mathrm{~m}} \mathrm{Tc}$-HMPAO, cerebral seizures 248

SPECT, versus PET, Editorial 2

Stomach emptying, dumping syndrome, transit time 195

${ }^{99 \mathrm{~m}} \mathbf{T c}$-aminohexylidendiphosphonate, myocardial infarction, experimental 226

${ }^{99 m}$ Tc-diphosponates, bone matrix, bone lesion detectability 8

${ }^{99 \mathrm{~m}}$ Tc-DTPA, renogram, deconvolution analysis 188

${ }^{99 \mathrm{~m}}$ Tc-HMPAO, cerebral seizures 248

${ }^{99 \mathrm{~m}} \mathrm{Tc}-\mathrm{HMPAO}$, fundamental and clinical research, reports 108

${ }^{99} \mathrm{~m}$ Tc-HMPAO, leukocytes, bone, inflammation 121 
${ }^{{ }^{* m} T c-H M P A O}$, leukocytes, ${ }^{9 \% m} \mathrm{Tc}-$ nanocolloid 63

${ }^{99 \mathrm{~m}}$ Tc-HMPAO, ${ }^{99 \mathrm{~m}} \mathrm{Tc}-\mathrm{RBG}$, cerebral perfusion reserve 46

${ }^{99 \mathrm{~m}} \mathrm{Tc}$-microspheres, liver, metastases, intraarterial cytostasis, control 228

${ }^{99 \mathrm{~m}}$ Tc-PMT, preparation of instant kit 154

${ }^{99 m}$ Tc-pyrophosphate, ${ }^{111}$ In-antimyosin, rhabdomyolysis, case report 38

${ }^{99 m}$ Tc-pyrophosphate, myocardial infarction, experimental 226
${ }^{99 m}$ Tc-sucralfate, ulcer detection 32

Thyroid carcinoma, follow-up, TSH sensitive 24

Thyroid carcinoma, metastatic, high-dose therapy concept 266

Thyroid, functional disorders, TSH basal and $\mathrm{T}_{3} 29$

$\mathrm{TSH}$, basal and $\mathrm{T}_{3}$, functional disorders of thyroid 29

TSH, sensitive, follow-up, thyroid carcinoma 24
Tube patency 252

Tumor, ${ }^{67} \mathrm{Ga}$-uptake, transferrin 151

$\mathrm{T}_{3}$, TSH basal, functional disorders of thyroid 29

Ulcer, detection, ${ }^{99 \mathrm{~m}} \mathrm{Tc}$-sucralfate 32

${ }^{133} \mathbf{X e - D S P E C T}$, cerebrovascular reserve capacity 127 


\section{Stichwortverzeichnis - Abstracts}

\section{Datenverarbeitung}

Hochauflösende PET $2 / 42$

SPECT, $180^{\circ}$ versus $360^{\circ} 2 / 43$

Computersimulation, Herzfunktion $2 / 43$

\section{Erythropoese}

Erythropoetin, Ferrokinetik 2/41

\section{First-Pass-Studie}

Rezirkulation, Abtrennung 2/50

\section{Gastroenterologie}

Magenresektion, Funktion 2/17

Ösophagus, Parameterbilder 2/17

Jejunoileostomie, Funktion 2/17

Sklerodermie, Funktionsszintigraphie $2 / 18$

Schilling-Test, B12 2/18

Leukozyten, entzündliche Darmerkrankungen $2 / 19$

Colitis ulcerosa, Granuloszint 2/19

Colitis ulcerosa, Granulozyten Ak 2/19

\section{Immunszintigraphie}

Ovarialkarzinom(e), anti-CA 125 2/8

Kolorektale Tumoren, anti-CA 19-9 2/8

Kombination, Applikationswege 2/9

Streptavidin-Biotin-System $2 / 9$

99m Tc-MAK, BW 431/26 2/29

${ }^{99 \mathrm{~m}} \mathrm{Tc}-\mathrm{MAK}$, intrazelluläres Verhalten $2 / 29$

${ }^{99 m}$ Tc-anti-CEA, colorektale Karzinome $2 / 30$

${ }^{99 \mathrm{~m}} \mathrm{Tc}$-anti-CEA, colorektale Tumorrezidive $2 / 30$

${ }^{99 \mathrm{~m}} \mathrm{Tc}$-anti-CEA, verschiedene Tumoren $2 / 30$

\section{Knochen}

Thyreoidektomie, Knochendichte $2 / 33$

Skelettszintigraphie, Traumatologie 2/33

Szintimetrie, Traumata $2 / 33$

Zementlose TEP, quantitativer Uptake $2 / 34$

Osteosarkom, COSS Studie 2/34

Mamma-Ca, MCA 2/34

Renale Osteopathie 2/42

\section{Leukozyten}

MAK BW 250/183 2/21

${ }^{99 \mathrm{~m}}$ Tc-MAK $2 / 21$

${ }^{99 \mathrm{~m}} \mathrm{Tc},{ }^{123} \mathrm{~J}-\mathrm{MAK} 2 / 21$

${ }^{123} \mathrm{~J}-\mathrm{MAK} 2 / 21$

${ }^{99 m}$ Tc-MAK, ${ }^{111}$ In-Oxin 2/22
${ }^{99 \mathrm{~m}}$ Tc-MAK, ${ }^{99 \mathrm{~m}}$ Tc-HMPAO 2/22

${ }^{99 m}$ Tc-MAK, Abszeß 2/23

${ }^{111}$ In, Hüft TEP $2 / 43$

\section{Myokard}

SPECT, Hinterwand 2/14

Stumme Ischämie $2 / 14$

Narbe, Kriterien 2/14

Rheologische Parameter 2/16

Linksschenkelblock 2/16

Fettsäuren, Oxidation 2/31

Fettsäuren, KHK 2/31

Fettsäuren, versus ${ }^{201} \mathrm{Tl}$ 2/31

Fettsäuren, Angioplastie 2/32

${ }^{201} \mathrm{Tl} /{ }^{99 \mathrm{~m}} \mathrm{Tc}-\mathrm{PPi}$, Nekrosen $2 / 32$

${ }^{81} \mathrm{Rb} /{ }^{81 \mathrm{~m}} \mathrm{Kr} 2 / 41$

\section{Nephro-Urologie}

Isotopenmiktionszysturographie $2 / 10$

Nierenfunktion, ESWL 2/10

Gadolinium-DTPA, NMR 2/12

Captopril, NAST $2 / 12$

Belastung, NAST $2 / 12$

${ }^{99 \mathrm{~m}}$ Tc-MAG3, Kinetik 2/13

${ }^{99 \mathrm{~m}}$ Tc-MAG3, Parameterbilder 2/13

${ }^{99 \mathrm{~m}}$ Tc-MAG3, klinische Ergebnisse 2/13

\section{NMR}

Linksventrikuläre Thromben $2 / 38$

Aortendissektion 2/38

Angioblastome 2/38

Laktatkonzentration $2 / 40$

Vorhofseptumdefekte $2 / 49$

Aorteninsuffizienz 2/49

\section{Onkologie}

Lymphszintigraphie, NMR 2/23

Lymphszintigraphie, malignes Melanom $2 / 23$

Knochenmarkszintigraphie, Neoplasien $2 / 24$

Knochenmarkszintigraphie, NMR, Plasmozytom $2 / 24$

${ }^{99 \mathrm{~m}} \mathrm{Tc}-\mathrm{MIBI},{ }^{201} \mathrm{Tl}$, Schilddrüsenkarzinom $2 / 24$

${ }^{123} \mathrm{~J}$-Methyltyrosin, Hirntumoren $2 / 25$

MIBG, Neuroblastom, Phäochromozytom, MEN II-Syndrom 2/25

MIBG, Sipple-Syndrom 2/26

\section{PET}

Lungentumoren $2 / 9$

FDG, Transportkonstanten in Tumoren $2 / 53$

Hirntumoren, Chemotherapie 2/53

Hirn, Proteinsynthese 2/54

\section{Radionuklidventrikulographie}

Intensivstation, mobile Kamera 2/47

Verlauf nach PTCA $2 / 47$

Adriamycin, Dosierung 2/48

${ }^{99 \mathrm{~m}} \mathrm{Tc}$-Isonitrile $2 / 48$

Akzessorische AV-Verbindungen 2/49

\section{Radiopharmakologie}

Ruthenocen-Amin-Derivate 2/40

${ }^{123} \mathrm{~J}$-Röntgenkontrastmittel 2/40

${ }^{99 \mathrm{~m}}$ Tc-HMPAO, Muskelperfusion 2/41

\section{Schilddrüse}

Ultraschall, Grauwertanalyse 2/44

TCTU, TSH, SD-Volumen 2/44

TCTU, regional $2 / 45$

Autonomie, ${ }^{201} \mathrm{Tl}$ 2/46

Hyperthyreose, PG-Bindungsstellen 2/46

Autonomie, definitive Therapie 2/47

Radiojodtherapie, Frühhypothyreose $2 / 50$

Veränderungen nach Bestrahlung 2/50

Onkozytäre SD-Ca, Radiojodspeicherung $2 / 52$

\section{Radiojodtherapie}

Dosierungsschema 2/52

Hormone, Schwangerschaft 2/52

\section{Therapie}

Immuntherapie, Radionuklide $2 / 35$

Immuntherapie, solitäre Lebermetastase $2 / 35$

Immuntherapie, Peritonealkarzinose 2/35

Malignes Phäochromozytom 2/36

M. Basedow, verschiedene $\cdot$ Herddosen 2/36

Radiojodtherapie, NMR, Knochenmarkszintigraphie $2 / 37$

Radiosynovektomie $2 / 37$

Radiojodtherapie, periphere Hormone $2 / 37$

\section{ZNS}

${ }^{133}$ Xe-DSPECT, Reservekapazität 2/26

${ }^{99 \mathrm{~m}} \mathrm{Tc}$-HMPAO, $\mathrm{CO}_{2}$-Spiegel $2 / 27$

Fluß-Volumen-SPECT $2 / 27$

${ }^{99 \mathrm{~m}}$ Tc-HMPAO, TCT $2 / 27$

${ }^{99 \mathrm{~m}}$ Tc-HMPAO, Epilepsie 2/28

${ }^{99 m}$ Tc-HMPAO, Demenz 2/28

Perfusion, Karotiskompression 2/28

Karotisstenose, ${ }^{99 \mathrm{~m}} \mathrm{Tc}-\mathrm{LDL},{ }^{111} \mathrm{In}$ Thrombozyten $2 / 44$ 


\section{K. Tatsch, P. Knesewitsch, C.-M. Kirsch, G. Küffer, G. Dörner, A. Matuschke*, J. Bogner*, F. D. Goebel*, E. Moser}

Aus der Radiologischen Klinik und Poliklinik

(Dir.: Prof. Dr. med. Dr. h. c. J. Lissner)

und der Medizinischen Poliklinik*

(Dir.: Prof. Dr. med. N. Zöllner)

der Universität München, BRD

Value of ${ }^{67} \mathrm{Ga}$ Scintigraphy in Primary Diagnosis and Follow-Up of Opportunistic Pneumonias in Patients with AIDS

\section{Stellenwert der ${ }^{67} \mathrm{Ga}-S z i n t i-$ graphie in der Primärdiagnostik und Verlaufsbeurteilung opportunistischer Pneumonien bei Patienten mit AIDS ${ }^{\star \star}$}

Opportunistic pneumonias are a life-threatening complication in patients with AIDS. Early diagnosis and therapy is necessary to improve prognosis. This study was designed to assess the value of ${ }^{67} \mathrm{Ga}$ scintigraphy in the primary detection and follow-up of these special pneumonias. ${ }^{67} \mathrm{Ga}$ scintigraphy was performed in 40 patients: 10 normal controls and 30 HIV-positive patients with AIDS or AIDS-related complex (ARC). ${ }^{67} \mathrm{Ga}$ scan results were compared with current chest radiographs and the results of pathogen detection. The evaluation of positive scans was based on a quantification of the pulmonary uptake, expressed as a pulmonary/soft-tissue uptake ratio. Only $8 / 30$ patients had a normal scan, $22 / 30$ showed diffuse (13/22) or focal $(9 / 22)$ increases of pulmonary uptake. In $7 / 8$ patients with normal scans the chest radiograph was negative as well. The one patient with negative scan but positive chest radiograph had pulmonary Kaposi's sarcoma. In 11/22 patients the ${ }^{67}$ Ga scan and chest radiograph were positive simultaneously. In the other 11/22 patients with positive scans chest radiographs were initially negative but showed pathology in 5 cases within 1-2 weeks. The reason for positive scans in most cases was an opportunistic lung infection; other forms of pneumonia were only observed in two cases. The defined uptake ratio demonstrated to be a highly sensitive parameter for monitoring pneumonia and the effects of therapy in follow-up studies. In conclusion, quantitative ${ }^{67} \mathrm{Ga}$ scintigraphy proved to be a reliable and highly sensitive method for primary detection and follow-up of opportunistic pneumonias in patients with AIDS.

\section{Zusammenfassung}

Opportunistische Pneumonien bedeuten für AIDS-Patienten eine lebensbedrohliche Komplikation. Zur Verbesserung der Prognose sind frühzeitige Diagnosestellung und Thera-

\footnotetext{
** Herrn Prof. Dr. Dr. h.c. J. Lissner zum 65. Geburstag gewidmet.
}

(Eingegangen: 19. Februar 1988; in revidierter Form: 16. Mai 1988) pieeinleitung unerläßlich. Ziel der vorliegenden Studie war es, den Stellenwert der ${ }^{67} \mathrm{Ga}$-Szintigraphie für Primärdiagnostik und Verlaufsbeurteilung dieser spezifischen Pneumonieformen $\mathrm{zu}$ untersuchen. Dic Suludie umfaßte 40 Patienten: 10 Lungengesunde und 30 HIV-positive Patienten mit AIDS oder AIDS-related complex (ARC). Die Befunde von Szintigraphie und konventionellem ThoraxRöntgen wurden verglichen und dem Erregerspektrum zugeordnet. Kriterium für ein pathologisches Szintigramm war ein am lungengesunden Normalkollektiv ermittelter Lunge/
Weichteilquotient (LWQ). Nur 8/30 Patienten hatten ein normales Szintigramm. 22/30 wiesen diffuse (13/22) oder fokale (9/22) Mehranreicherungen in der Lunge auf. 7/8 Patienten mit normalem Szintigramm waren gleichzeitig Röntgen-negativ. In dem Einzelfall mit positivem Röntgenbefund und negativem Szintigramm lag ein pulmonales Kaposi-Sarkom vor. Bei 11/22 Patienten mit positivem Szintigramm war gleichzeitig der Röntgenbefund pathologisch. Die anderen 11 Patienten mit pathologischem Szintigramm hatten einen negativen Röntgenausgangsbefund, der 
aber in 5 Fällen noch innerhalb 1-2 Wochen positiv wurde. Pathologische Szintigramme waren meist Folge einer opportunistischen Pneumonie; gewöhnliche Pneumonien kamen selten vor. Zur Therapiekontrolle und Verlaufsbeurteilung opportunistischer Pneumonien sind als hochsensitiver und objektivierbarer Parameter ${ }^{67} \mathrm{Ga}$ Verlaufsuntersuchungen mit Quantifizierung der Lungenanreicherung zu empfehlen.

\section{Einleitung}

Für Patienten mit AIDS bedeuten opportunistische Infektionskrankheiten eine lebensbedrohliche Komplikation. Wie die letzten Jahre gezeigt haben, wird die Prognose quoad vitam dabei in erster Linie von Erkrankungen der Lunge bestimmt $(9,21,26)$. Andere Prädilektionsstellen opportunistischer Infektionen, wie z.B. der Gastrointestinaltrakt, haben vergleichsweise nur eine geringere $\mathrm{Be}$ deutung (27).

Opportunistische Pneumonien werden bei AIDS-Patienten am häufigsten durch den Parasiten Pneumocystis carinii $(2,9,22,26)$, atypische Mykobakterien $(12,18)$ oder das Cytomegalievirus (20) verursacht. Die hohen Morbiditäts- und Letalitätsraten $(6,21)$, insbesondere bei der Pneumocystis-carinii-Pneumonie, belegen die Notwendigkeit, durch frühzeitige Diagnosestellung und Einleitung einer spezifischen Therapie die Prognose der Patienten zu verbessern. Da subjektives Beschwerdebild und klinischer Untersuchungsbefund im Frühstadium der Pneumonie jedoch häufig nur diskrete Hinweise liefern $(2,12$, 14, 21), kann die Diagnosestellung erhebliche Schwierigkeiten bereiten.

Die vorliegende Studie untersucht, welchen Stellenwert die ${ }^{67} \mathrm{Ga}$-Szintigraphie für die Primärdiagnostik und Verlaufsbeurteilung dieser besonderen Pneumonieformen besitzt. Dazu werden die Ergebnisse der Szintigraphie mit der konventionellen ThoraxRöntgendiagnostik und den klinischen Befunden korreliert.

\section{Patienten und Methodik}

Insgesamt wurden 40 Patienten untersucht 10 HIV-negative Personen ohne Lungenerkrankung stellten für die szintigraphischen Untersuchungen das Normalkollektiv. Bei 30 Patienten mit serologisch gesichertem HIV-Nachweis wurde neben der Standard-Diagnostik auch ei ne ${ }^{67} \mathrm{Ga}$-Szintigraphie durchgeführt. Ein Großteil der Patienten wies zum Zeitpunkt der Untersuchung klinisch unspezifische Symptome wie Fieber, Husten oder Dyspnoe auf. Die Patienten waren zwischen 24 und 50 Jahre alt bei einem Medianwert von 33 Jahren. Mit 28 Männern bei nur 2 Frauen war das Verhältnis deutlich zu Lasten des männlichen Geschlechts verschoben.

Die Klassifikation der Patienten erfolgte entsprechend den Kriterien der Centers for Disease Control (7). Bei 22 Patienten lag demnach AIDS im Stadium der klinischen Manifestation vor, 8 Patienten hatten zum Zeitpunk der Untersuchung noch die Diagnose AIDSrelated complex (ARC), ein Vorstadium der klinisch manifesten Erkrankung.

Zur Durchführung der Szintigraphie wurde den Patienten $185 \mathrm{MBq}{ }^{67} \mathrm{Ga}$-Zitrat intravenös verabreicht. Die Szintigraphie wurde aus schließlich in planarer Technik durchgeführt. 48 und $72 \mathrm{~h}$ nach Injektion erfolgten Lungenaufnahmen von ventral und dorsal. Für die Aufnahmen wurde eine Großfeldgammakamera (mittelenergetischer Kollimator, 1 Peak-Messung bei $93 \mathrm{keV}$ ) mit angeschlossenem Rechnersystem verwendet: Die Aufnahmezeit pro Bild betrug $10(24 \mathrm{~h})$ bzw. $15(72 \mathrm{~h}) \mathrm{min}$. Im Durchschnitt lagen dabei 1,5-2 Millionen counts pro Bild vor.

Zusätzlich zu der rein visuellen Bildinterpretation wurde als Grundlage für eine objektive Wertung von Ausgangs- und Verlaufsbefunden in den Aufnahmen nach $72 \mathrm{~h}$ die Lungenanreicherung mittels ROI-Technik quantifiziert. Aus dem Produkt von ventraler und dorsaler Zählratendichte wurde die Wurzel gezogen. Dieses geometrische Mittel galt als ${ }^{67} \mathrm{Ga}-$ Lungen-Uptake. Auf identische Weise wurde die Ga-Anreicherung im lateralen Halsweichteilmantel (unter Aussparung der HWS) quantifiziert und als Bezugsgröße herangezogen. Der Quotient aus ${ }^{67}$ Ga-Lungen-Uptake und Weichteilspeicherung wird im folgenden mit LWQ bezeichnet und ist ein Parameter für inter- und intraindividuelle Vergleiche. Die diagnostische Entscheidung - normal oder pathologisch - erfolgte lediglich an den LWQ-Werten.

Das Ergebnis der Ga-Szintigraphie wurde jeweils mit dem aktuellen Thorax-Röntgenbefund (zeitlicher Abstand zur Szintigraphie maximal 4 Tage) und den klinischen Angaben bzw. Befunden (Bronchoskopie mit Lavage und/oder Gewebsgewinnung, mikrobiologischer Erregernachweis) korreliert. Von 8 Patienten lagen zusätzlich zu dem Ausgangsszintigramm Verlaufsuntersuchungen mit ${ }^{67} \mathrm{Ga}$ vor. Das Intervall zwischen den Untersuchungen betrug wenigstens 6 Wochen und längstens 6 Monate, im Mittel waren es 2 Monate.

\section{Ergebnisse}

\section{Ergebnisse der ${ }^{67} \mathrm{Ga}$-Szintigraphie}

Befunde bei Lungengesunden. Im lungengesunden Kontrollkollektiv unterschied sich die Lungenanreicherung nicht von der des umgebenden Weichteilgewebes. Dies äußerte sich quantitativ in einem LWQ, der im Mittel bei $0,95 \pm 0,05$ SD lag. In Anlehnung an diese Befunde wurde in der vorliegenden Untersuchung ein LWQ $<1,1$ (Mittelwert +3 SD) als normal definiert, ein LWQ $>1,1$ galt als pathologisch und wies auf eine erhöhte GaAnreicherung der Lungen hin.

Befunde bei AIDS-Patienten. Bei 8/ 30 Patienten $(27 \%)$ wurde im Ausgangsszintigramm ein Normalbefund erhoben. Darunter waren je $4 \mathrm{mit}$ Diagnose ARC oder AIDS. In den Fällen mit normalem Szintigramm (Abb. 1a) lagen LWQ-Werte im Bereich von 0,88 bis 1,00 (Mittelwert: $0,95 \pm 0,04)$ vor. Damit war in jedem Einzelfall der LWQ unter dem Grenzwert von 1,1

Ein erhöhter LWQ im Ausgangsszintigramm wurde bei 22 Patienten $(73 \%)$ beobachtet, darunter waren 17 mit AIDS und 5 mit ARC. Die LWQWerte lagen im Bereich zwischen 1,25 und 2,63 (Mittelwert: 1,51 $\pm 0,28$ ) und damit stets über dem definierten Grenzwert von 1,1. Szintigraphisch waren zwei pathologische Anreicherungsformen zu unterscheiden: Bei 13 Patienten lag eine diffuse Mehrspeicherung in beiden Lungenhälften vor (Abb. 1b). In 9 Fällen war die pathologische Mehranreicherung fokal begrenzt. Parahiläre, zentrale und basale Lungenanteile (Abb. 1c) waren bevorzugt befallen, fokale Mehrspeicherungen in den Lungenoberfeldern wurden nie beobachtet. In zwei Fällen mit parahilärer Speicherung ließ sich die Anreicherung in Lymphknoten lokalisieren, in den übrigen Fällen war mit planarer Aufnahmetechnik keine Abgrenzung von Lymphknoten und Lungenparenchym möglich.

\section{Korrelation der Befunde von Szintigraphie und Thorax-Röntgen}

Neben unauffälligen Befunden umfaßte das Spektrum röntgenologischer 
Veränderungen in der Thoraxaufnahme in erster Linie interstitielle Infiltrate in retikulärer, retikulonodulärer oder nodulärer Form. Die Befunde

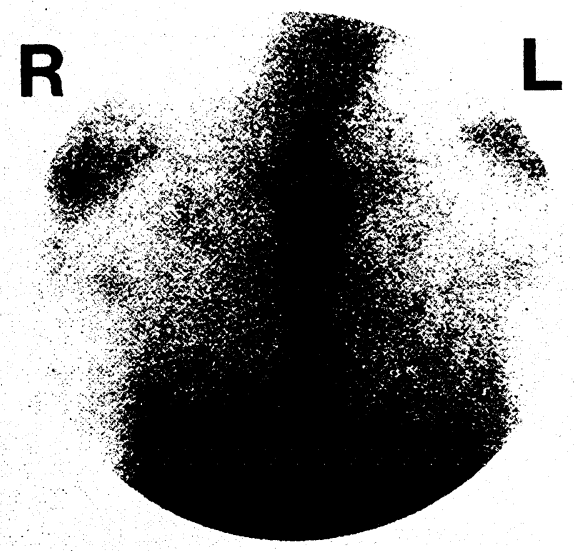

a

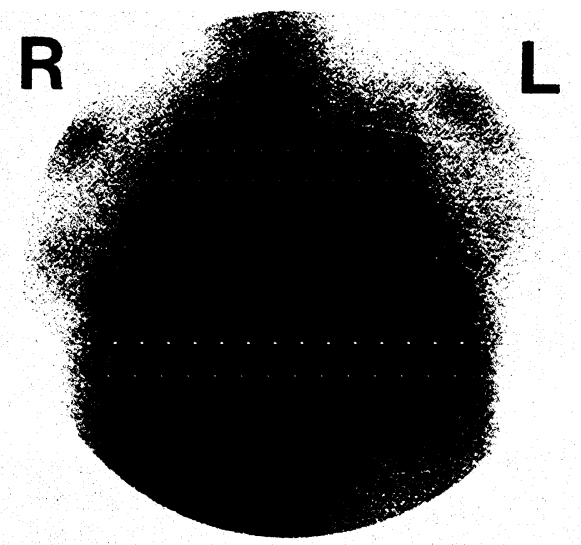

b

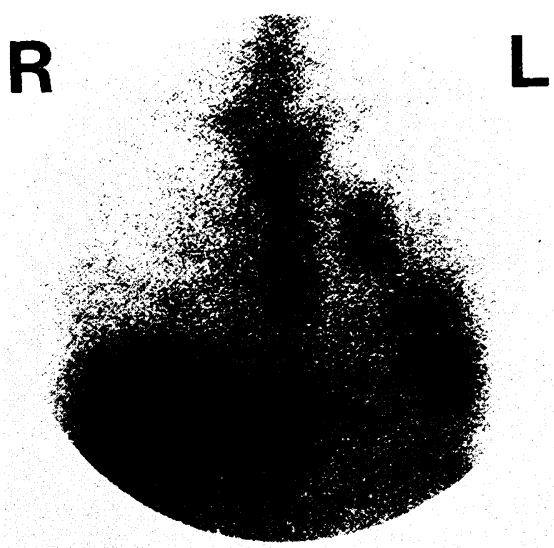

c

$A b b .1$ Anreicherungsmuster im ${ }^{67} \mathrm{Ga}$-Szintigramm bei AIDS-Patienten: a) Normalbefund (LWQ 1,0), b) pathologische, diffuse Mehranreicherung beider Lungen (LWQ 2,3), c) pathologisch erhöhte Aktivitätsbelegung in fokaler Form (LWQ 1,8). reichten bis zu einer milchglasartigen Trübung der gesamten Lunge. Auf exakte zahlenmäßige Aufschlüsselung der verschiedenen Infiltratformen wurde verzichtet, da das Überwiegen von Mischformen eine exakte Klassifizierung erschwerte. Eine alveoläre Infiltration wurde nur selten beobachtet. Veränderungen im Röntgenbild nahmen typischerweise ihren Ausgang vom Hilusbereich und betrafen in erster Linie den Lungenkern. Eine Ausbreitung fand, falls nachweisbar, in zentrifugaler Richtung statt.

Das Ergebnis der Korrelation der Befunde von Szintigraphie und Thorax-Röntgen ist als Vierfeldertafel in Tab. 1 zusammengefaßt. In 7 Fällen mit unauffälligem Röntgenbild bei normalem Szintigramm bestätigte der blande klinische Verlauf in der Folgezeit die Befunde als richtig negativ. Ein AIDS-Patient zeigte bei negativem Szintigramm (Abb.2a) einen positiven Röntgenbefund (Abb.2b). Das negative Szintigramm sprach gegen das Vorliegen eines floriden Entzündungsprozesses und stimmte bei diesem Patienten mit der subjektiven Symptomfreiheit und dem klinisch unauffälligen Untersuchungsbefund überein. Ursache des positiven Röntgenbefundes war ein pulmonales $\mathrm{Ka}$ posi-Sarkom, das bronchoskopisch zu sichern war.

11 Patienten zeigten bei der Ausgangsuntersuchung einen pathologischen szintigraphischen und röntgenologischen Befund. In 7 Fällen stimmten Lokalisation und Ausmaß der Befunde überein, in den übrigen 4 Fällen war im Szintigramm der Lungenbefall ausgedehnter als im Röntgenbild.

Pathologische Mehranreicherungen im Szintigramm bei normalem Röntgenbefund wurden bei 11 Patienten beobachtet (Abb. 3a, b). Eine Abhängigkeit dieser Befunde vom szintigraphischen Speicherungsmuster (fokal; diffus) war nicht gegeben. Verlaufsaufnahmen zeigten, daß bei 5 der 11 Patienten das zunächst negative Röntgenbild später doch Infiltrate zeigte. Dieser Befund war im Mittel 10 Tage (Bereich: 4-15 Tage) nach dem positiven Szintigramm zu dokumentieren (Abb. 3c).
Erregerspektrum bei positiven $G a-$ Szintigrammen

In 20/22 Fällen mit positivem Szintigramm konnten Pneumonieerreger durch Bronchiallavage, bronchoskopisch gewonnenes Material und Kulturen nachgewiesen werden. Tab. $2 \mathrm{faßt}$ die Ergebnisse zusammen. In der Mehrzahl der Fälle lag der Anreicherung im Szintigramm eine Pneumonie durch opportunistische Erreger zu-

Tab. 1 Korrelation der Befunde von Szintigraphie und Thorax-Röntgen.

\begin{tabular}{llll}
\hline & & \multicolumn{2}{c}{ Szintigramm } \\
& & negativ & positiv \\
\hline Thorax- & negativ & 7 & 11 \\
Röntgen & positiv & 1 & 11 \\
\hline
\end{tabular}

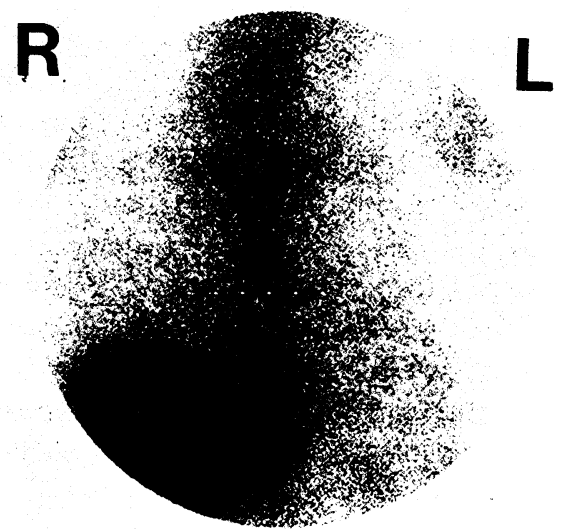

a
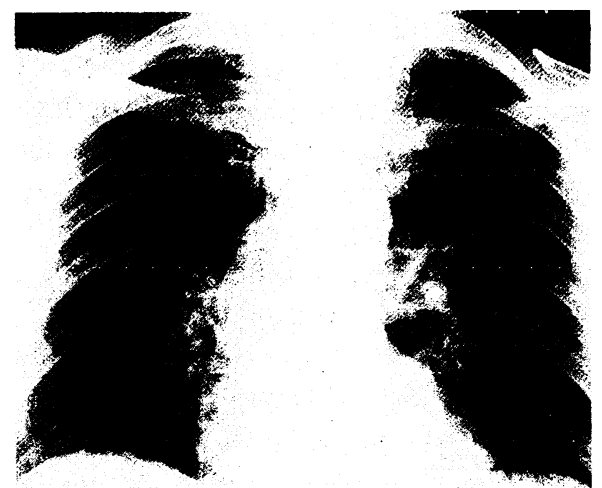

b

Abb. 2 AIDS-Patient mit pulmonalem Kaposi-Sarkom: a) Normalbefund im ${ }^{67} \mathrm{Ga}$-Szintigramm (LWQ 0,9), b) pathologischer Röntgenbefund. 
grunde $(n=18)$. Am häufigsten wurde Pneumocystis carinii als Erreger gesichert, gefolgt von atypischen Mykobakterien (M. avium, M. xeno-

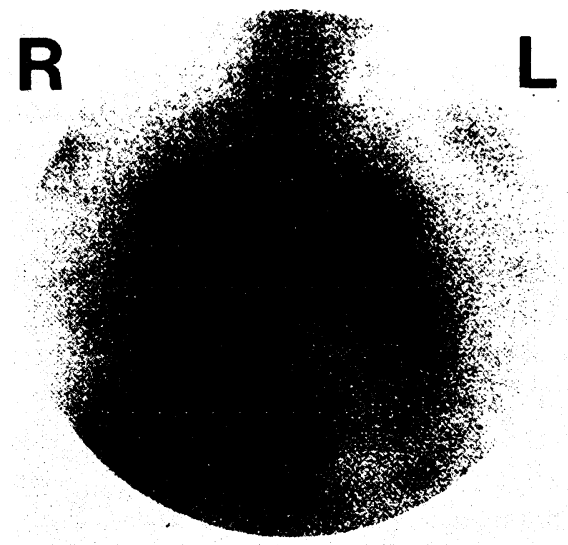

a)


b)

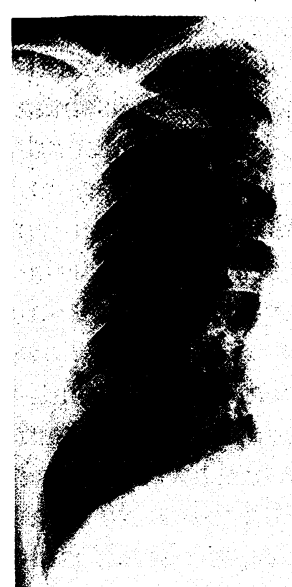

c)

Abb. 3 AIDS-Patient mit einer Pneumocystiscarinii-Pneumonie: a) Hochgradige diffuse Mehranreicherung in beiden Lungen (LWQ $2,1)$, b) unauffälliger Röntgenbefund zum Zeitpunkt der Szintigraphie, c) pathologischer Röntgenbefund 10 Tage später. Szintigrammen. pi, M. fortuitum). Eine CytomegaliePneumonie konnte in unserem Kollektiv nur selten nachgewiesen werden. In einem Fall lag gesichert eine Mischinfektion von Pneumocystis carinii mit Mykobakterium avium vor.

Tab. 2 Erregerspektrum bei positiven ${ }^{67} \mathrm{Ga}$ -

\begin{tabular}{ll}
\hline Opportunistische Infektionen & $\mathrm{n}=18$ \\
Pneumocystis carinii & $\mathrm{n}=11$ \\
Atypische Mykobakterien & $\mathrm{n}=4$ \\
Cytomegalie & $\mathrm{n}=2$ \\
Pneum. carinii/atyp. Mykobakt. & $\mathrm{n}=1$ \\
Bakterielle Infektionen & $\mathrm{n}=2$ \\
Kein Erregernachweis (bisher) & $\mathrm{n}=2$ \\
\hline
\end{tabular}

Der Zusammenhang zwischen Anreicherungsmuster und Erregerspektrum ist in Tab. 3 dargestellt. Bei mittel- bis hochgradiger diffuser Anreicherung im Szintigramm (LWQ $>1,6)$ wurde stets Pneumocystis carinii als Erreger nachgewiesen, derselbe Keim war allerdings auch in Fällen mit fokaler Mehrspeicherung als Pneumonieerreger nachzuweisen. Pneumonien durch atypische Mykobakterien zeigten stets Anreicherungen in fokaler Form.

In 2 Fällen waren bakterielle Infektionen (Streptococcus pneumoniae, Staphylococcus aureus) für einen fo-

Tab. 3 Zusammenhang zwischen Anreicherungsmuster und Erregerspektrum.

Cytomegalie kein Erreger $\quad(\mathrm{n}=13)$

Tab. 4 Ergebnisse der szintigraphischen Verlaufsuntersuchungen.

\begin{tabular}{|c|c|c|c|c|c|c|}
\hline $\mathrm{N}$ & & LWQ & & Anreicherung & Erreger & Befund \\
\hline 1 & 1,4 & 1,8 & 2,1 & fokal & Atyp. Mykobakt. & Progression \\
\hline 2 & 1,5 & 1,7 & 1,5 & fokal & Pneum. carinii & $\varnothing$ Ânderung \\
\hline 3 & 1,5 & 1,4 & 1,4 & fokal & Atyp. Mykobakt. & $\varnothing$ Änderung \\
\hline 4 & 2,3 & 1,6 & & diffus & Pneum. carinii & Regression \\
\hline 5 & 1,5 & 1,4 & 1,2 & fokal & Atyp. Mykobakt. & Regression \\
\hline 6 & 1,6 & 1,0 & & diffus & Pneum. carinii & Normalisierung \\
\hline 7 & 1,5 & 1,1 & 1,0 & diffus & Pneum. carinii & Normalisierung \\
\hline 8 & 2,0 & 1,4 & 0,9 & diffus & Pneum. carinii & Normalisierung \\
\hline
\end{tabular}

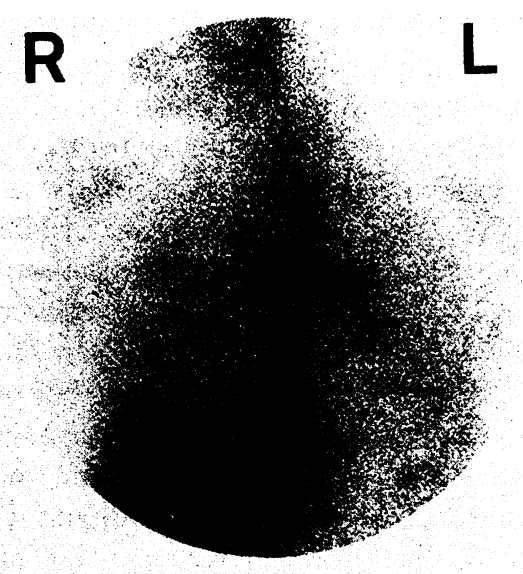

a)

$A b b .4$ AIDS-Patient mit einer Pneumonie durch atypische Mykobakterien. Befundprogredienz in der Verlaufsuntersuchung: a) Im Ausgangsbefund fokale Mehranreicherung beidseits parahilär (LWQ 1,4), b) deutliche Zunahme der fokalen Mehranreicherung in bezug auf Intensität und Ausdehnung des Befundes 4 Monate später. 


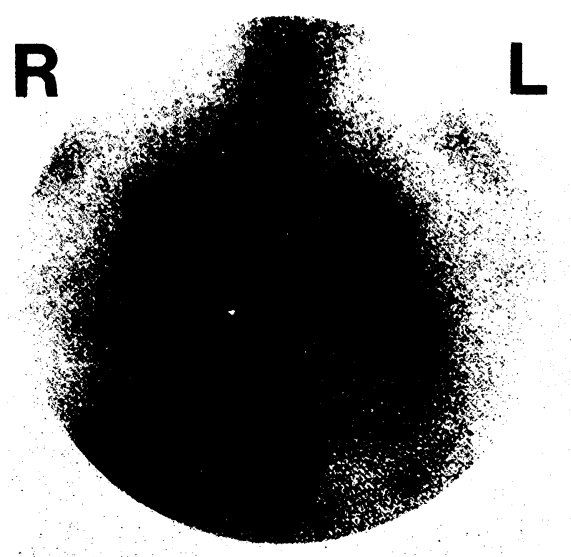

a)

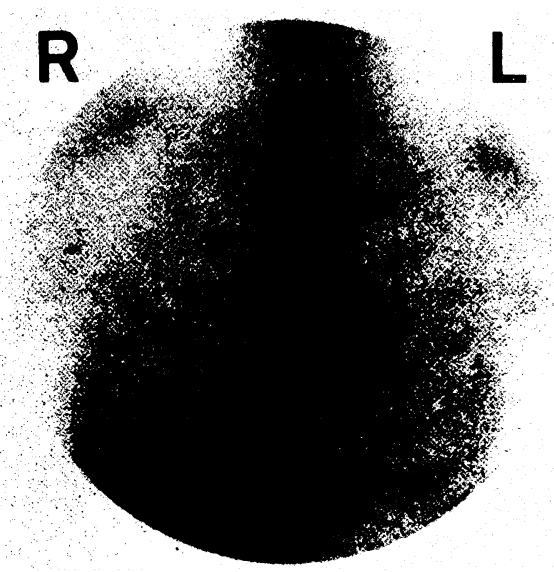

b)

$A b b .5$ AlDS-Patient mit einer Pneumocystiscarinii-Pneumonie. Nachweis der Befundnormalisierung durch szintigraphische Verlaufsuntersuchungen: a) Floride Pneumonie mit hochgradiger diffuser Mehranreicherung im Szintigramm (LWQ 1,6), b) normale Aktivitätsbelegung 3 Monate später (LWQ 1,0).

kal positiven Befund im Szintigramm verantwortlich. Zweimal blieb der Erregernachweis bislang negativ.

\section{Szintigraphische Verlaufsuntersuchungen}

Bei 8 Patienten lagen Verlaufsuntersuchungen vor. Als Parameter für eine untersucherunabhängige Beurteilung des Pneumonieverlaufs wurde der LWQ herangezogen, Tab. 4 faßt die Ergebnisse zusammen. Mit der Quantifizierung waren Befundprogredienz oder -regression im Pneumonieverlauf zuverlässig $\mathrm{zu}$ erkennen. Abb. 4 zeigt Fall 1 aus Tab. 4 als Beispiel einer Befundprogredienz, in Ab. 5 ist Fall 6 mit Befundnormalisierung bei einer Pneumonie dargestellt.

\section{Diskussion}

AIDS-Patienten werden durch opportunistische Infektionen lebensbedrohlich gefährdet $(9,21,26,27)$. Wie bei anderen Krankheitszuständen, die mit einer Suppression des Immunsystems verbunden sind, stellt auch bei AIDS die Lunge einen Hauptmanifestationsort opportunistischer Infektionen dar (21). Die Inzidenz dieser Pneumonien ist hoch; allein für die Pneumocystis-carinii-Pneumonie wurden Werte bei $60 \%$ gefunden $(6,9)$. Die Letalität wird im Bereich von $50 \%$ und mehr angegeben $(6,24,26)$. Diese Daten erfordern vermehrte Anstrengungen bei Diagnostik und Therapie AIDS-bedingter Infektionen. Gezielte therapeutische Maßnahmen setzen eine funktionsmorphologische und bakteriologische Diagnosesicherung voraus, da eine prophylaktische Medikation aufgrund hoher Nebenwirkungsraten (9) nicht generell in Frage kommt und die Behandlungsstrategie vom Erregertyp abhängt. Es ist allgemein akzeptiert, daß eine frühzeitige Diagnosestellung einer opportunistischen Infektion die Prognose entscheidend verbessert $(9,12,22)$. In dieser Frühphase sind die subjektiven Beschwerden jedoch gering und der klinische Untersuchungsbefund oft nicht ergiebig $(12,14,20)$.

Derzeit wird der Weg zur Diagnosesicherung noch kontrovers diskutiert. Zahlreiche Studien betonen den hohen Stellenwert der Bronchoskopie mit bronchoalveolärer Lavage oder Gewebsgewinnung, insbesondere zum Nachweis der häufigsten opportunistischen Pneumonie (Pneumocystis carinii) $(8,20,24)$. Die Methode ist allerdings invasiv, nicht ohne Komplikationen (20) und als Screening-Verfahren ungeeignet. Der konventionellen Thorax-Röntgenaufnahme kommt bei positiven Befunden Bedeutung zu, negative Befunde jedoch schließen, wie eine Reihe von Untersuchungen (2, $12,17,19,23,27)$ bereits gezeigt hat, eine opportunistische Infektion nicht aus. Schließlich wurde in der GaSzintigraphie, die seit langem neben der Tumordiagnostik auch als sensitives Verfahren für den Nachweis ent- zündlicher Prozesse eingesetzt wird $(4,11)$, eine erfolgversprechende Methode für die Diagnostik opportunistischer Pneumonien gesehen.

Erste Kasuistiken von Levenson et al. (14), gefolgt von Beiträgen anderer Untersucher $(15,25)$, zeigten an Patienten mit Pneumocystis-cariniiPneumonie bei unterschiedlichen Grunderkrankungen (Lymphom, Verdacht auf AIDS bei Homosexualität) die Überlegenheit der ${ }^{67} \mathrm{Ga}$-Szintigraphie im Vergleich zum Röntgenbild. Weitere Studien an begrenzten selektionierten Kollektiven $(2,12,27)$ stützen diese ersten kasuistischen Daten.

Die hier vorgelegten Ergebnisse bestätigen, daß die Ga-Szintigraphie ein hochsensitives Verfahren zum Nachweis entzündlicher Lungenveränderungen bei AIDS darstellt. In dem untersuchten Kollektiv war kein falsch negativer Befund nachweisbar (Sensitivität: $100 \%$ ). In der Literatur beziehen sich Angaben zur Sensitivität der ${ }^{67} \mathrm{Ga}$-Szintigraphie häufig nur auf die Pneumocystis-carinii-Pneumonie. In Abhängigkeit unterschiedlicher Untersucher und Wertungskriterien liegen die Angaben dazu zwischen 90\% und $100 \%(2,8,12,19,27)$. Nach derzeitigem Kenntnisstand spricht umgekehrt ein negatives Szintigramm gegen eine AIDS-Pneumonie. Kramer et al. (12) schreiben der Kombination unauffälliges Röntgenbild mit negativem ${ }^{67} \mathrm{Ga}$-Szintigramm einen hohen negativen Voraussagewert für einen pathologischen Lungenproze $B$ zu und betonen, daß ein negatives Szintigramm selbst bei pathologischem Röntgenbefund gegen einen entzündlichen Prozeß in der Lunge spricht. Mit der Einschränkung der kleinen eigenen Fallzahl waren diese Aussagen anhand der eigenen Beobachtungen zu bestätigen.

Angaben zur Spezifität der Szintigraphie sind problematisch, da zum sicheren Krankheitsnachweis eine komplexe und zum Teil invasive Diagnostik erforderlich ist, die im Einzelfall nur selten verantwortet werden kann. In dieser Studie blieben $2 \mathrm{~Pa}-$ tienten mit positivem Szintigramm ohne Erregernachweis, wobei aber bislang auf eine invasive Diagnostik verzichtet wurde. Unter diesem Vorbehalt betrug die Spezifität in der vorlie- 
genden Untersuchung $91 \%$. Angaben aus dem Schrifttum reichen von $51 \%$ bis über $90 \%(2,8,12,19)$, wobei für die Streuung unterschiedliche Untersuchungszeitpunkte und Wertungskriterien verantwortlich gemacht werden (12). Der Ergebnisvergleich von Szintigraphie und Röntgen zeigt entscheidende Unterschiede im Aussagewert beider Verfahren. 11/22 Patienten $(50 \%)$ mit positivem Szintigramm und gesichertem Erregernachweis wiesen gleichzeitig einen unauffälligen Thorax-Röntgenbefund auf. Dieses Ergebnis belegt die höhere Sensitivität der Szintigraphie im Vergleich zum Röntgen beim Nachweis opportunistischer Pneumonien. Negative Röntgenbefunde traten unabhängig vom szintigraphischen Anreicherungsmuster und Erregertyp auf. Bei 5 der 11 Patienten wurde der anfänglich negative Röntgenbefund mit einer zeitlichen Verzögerung von 1-2 Wochen positiv. Als Folgerung ergibt sich, daß opportunistische Pneumonien sich im Szintigramm frühzeitiger darstellen und damit eher behandelt werden können.

Bei einem Vergleich der Befundausdehnung fällt auf, daß bei 4 von 11 Patienten der szintigraphisch nachweisbare Befall erheblich umfangreicher war als im Röntgenbild. Die vorliegenden Ergebnisse bestätigen damit, daß, wie im Schrifttum (10) erwähnt, die Ausdehnung einer AIDS-Pneumonie im Röntgenbild allein unterschätzt werden kann. Gegen eine Überschätzung des szintigraphischen Befundes sprechen die pathophysiologischen Mechanismen (4) der Ga-Akkumulation im Entzündungsprozeß. Sie beruhen, soweit bekannt, auf einer erhöhten Gefäßpermeabilität, der direkten Aufnahme in Leukozyten und Mikroorganismen sowie einer hohen Affinität von Gallium zu dem Entzündungsmediator Laktoferrin. Diese Faktoren sind auf den Ort der Entzündung begrenzt. Zum Zeitpunkt der Szintigraphie ( $72 \mathrm{~h}$ p.i.) kann die Blutpoolaktivität als Ursache für falsch positive Befunde vernachlässigt werden.

In der Diagnostik des Kaposi-Sarkoms ist die ${ }^{67} \mathrm{Ga}$-Szintigraphie nur von untergeordneter Bedeutung. In einem kasuistischen Beitrag wiesen
Krishnamurthy et al. (13) bereits 1976 auf die fehlende ${ }^{67} \mathrm{Ga}$-Speicherung beim Kaposi-Sarkom hin. Diese Befunde wurden durch neuere Beiträge an AIDS-Patienten bestätigt $(12,19$, 27). Auch bei dem eigenen Patienten mit gesichertem pulmonalem KaposiSarkom war keine pathologische Anreicherung nachweisbar, der Röntgenbefund dagegen war positiv. Diese Befundkonstellation erhärtet bei Vorliegen von AIDS den Verdacht eines pulmonalen Kaposi-Sarkoms.

Eine Zuordnung von szintigraphischen Speicherungsmustern zum Erregertyp erlaubt folgende Aussagen:

Wie aus der Literatur bekannt (2, $8,12,19,27)$, weist eine hochgradige diffuse Anreicherung der gesamten Lunge mit hoher Wahrscheinlichkeit auf eine Pneumocystis-carinii-Pneumonie hin. Die eigenen Ergebnisse können weiterhin zeigen, daß die Angabe eines relativen Uptake-Parameters die Abgrenzung der Erreger verbessert. Bei einem LWQ >1,6 war ausschließlich Pneumocystis carinii nachweisbar. Ähnliche Ergebnisse werden in der Literatur auch für semiquantitative Gewichtungskonzepte der Anreicherungsintensität beschrieben, wobei die Speicherung von Leber, Knochenmark und Weichteilgewebe als Bezugsgrößen gewählt wurde. Auf mögliche Nachteile dieser Bezugsgrößen wird später gesondert eingegangen. Zusätzlich konnte gezeigt werden, daß atypische Mykobakterien ausschließlich einen fokalen Befall im Szintigramm verursachten. Kasuistische Beiträge von Malhotra et al. (16), Bach et al. (1) und Skarzynski et al. (18) wurden damit durch eigene Beobachtungen bestätigt. Die vorgelegten Ergebnisse zeigen darüber hinaus, daß das fokale Muster aber auch bei gewöhnlichen bakteriellen Pneumonien und vereinzelt bei Pneumocystis carinii nachweisbar war und schränkt damit die Spezifität einer fokalen Anreicherung im Hinblick auf einen bestimmten Erregertyp ein.

Wie Bitran et al. (3) bereits zeigten, kommt einem positiven Szintigramm bei Patienten mit ARC besondere Bedeutung zu, da mit dem Nachweis opportunistischer Pneumonieerreger definitionsgemäß AIDS im Stadium der klinischen Manifestation vorliegt.
Von einer derartigen Umklassifizierung mit prognostischer Bedeutung waren auch in der eigenen Studie 4 Patienten betroffen.

Ein positives Szintigramm war in der Regel Anlaß zur Bronchoskopie. Während diffuse Mehrspeicherungen keine Zusatzinformation liefern, haben sich fokale Mehrspeicherungen als wichtige Lokalisationshilfe für eine gezielte Bronchiallavage erwiesen.

Die durchgeführte Quantifizierung erweist sich zur Verlaufsbeurteilung hilfreich. Der LWQ-Parameter erleichtert die Beurteilung von Längsschnittstudien. Als Bezugsgröße wurde der Halsweichteilmantel gewählt. Dieser hat im Vergleich zu Leber und Knochenmark den Vorzug, daß die Anreicherung hier nicht zahlreichen und im Einzelfall schwer abschätzbaren Variablen unterliegt. An solchen sind für die Leber bekannt: Beeinflussung durch Medikamente, Drogen, Infektionen, Abhängigkeit der Speicherung von der hepatischen Enzymaktivität und der Anreicherung in anderen Organen. Für das Knochenmark ist noch ungeklärt, ob nicht AIDS zu einem veränderten Speicherverhalten führt. Dieser Aspekt gewinnt insbesondere dadurch an Bedeutung, als mit der Knochenmarkszintigraphie bereits eine hohe Inzidenz von Knochenmarkveränderungen bei diesem Patientenkollektiv nachgewiesen wurde (5). Aufgrund der aufgeführten Unwägbarkeiten sollte der Quantifizierung einer Anreicherung bezogen auf normales Weichteilgewebe gegenüber anderen Gewichtungskonzepten mit Bezug der Anreicherung auf Leber und Knochenmark der Vorzug gegeben werden. Die Wahl des lateralen Halsweichteilgewebes als Bezugsgröße erfolgte aus Praktikabilitätsgründen, da dieser Bereich in allen Aufnahmen miterfaßt war und sich Zusatzaufnahmen erübrigten.

Die vorgelegten Ergebnisse belegen, daß szintigraphische Untersuchungen mit Berechnung des LWQ hervorragend zur Verlaufsbeurteilung geeignet sind. Das Verfahren ist praktikabel, der Aufwand vertretbar. Un- 
ter dem Gesichtspunkt der Prognose von AIDS-Patienten kann auch die Strahlenexposition akzeptiert werden (ca. 5 rad für das untere Kolon).

\section{LITERATUR}

1 Bach M C, Bagwell S P, Masur H. Utility of gallium imaging in the diagnosis of Mycobacterium avium - intracellular infection in patients with the acquired immunodeficiency syndrome. Clin Nucl Med 1985; 11 : 175-7.

2 Barron T F, Birnbaum N S, Shane L B, Goldsmith S J, Rosen M J. Pneumocystis carinii pneumonia studied by gallium- 67 scanning. Radiology 1985; 154: 791-3.

3 Bitran J, Bekerman C, Weinstein R, Bennett $\mathrm{Ch}$, Ryo $\mathrm{U}$, Pinsky $\mathrm{S}$. Patterns of gallium-67 scintigraphy in patients with acquired immunodeficiency syndrome and the AIDS related complex. J Nucl Med 1987; 28: 1103-6.

4 Botsch H. Galliumszintigraphie: Diagnostik bei entzündlichen Erkrankungen und Tumoren. Berlin - Heidelberg - New York - Tokyo: Springer, 1985.

5 Brandhorst I, Ganser A, Brodt H-R et al. Scintigraphic bone marrow status in acquired immunodeficiency syndrome (AIDS): First results in 22 patients. Nuklearmedizin. Stuttgart - New York: F. K. Schattauer, 1986; 654-6.

6 Centers for Disease Control Update: Acquired immunodeficiency syndrome (AIDS) - United States. Morbid Mortal Weekly Rep 1983; 32: 309-11.

7 Centers for Disease Control Update: Acquired immunodeficiency syndrome (AIDS) - United States. Morbid Mortal Weekly Rep 1984; 32: 691.

8 Coleman D L, Hattner R S, Luce J M, Dodek P M, Golden J A, Murray J F. Correlation between gallium lung scans and fiberoptic bronchoscopy in patients with suspected pneumocystis carinii pneumonia and the acquired immune deficiency syn- drome. Am Rev Respir Dis 1984; 130: 1166-9.

9 Goebel F D, Zoller W G. Pneumocystis carinii-Pneumonie bei Patienten mit erworbenem Immundefekt-Syndrom (AIDS). AIDS-Forsch 1986; 2: 89-96.

10 Graybeal F R, Berens S V, Jurgensen P F. Gallium-67 scintigraphy in acquired immune deficiency syndrome complicated by pneumocystis carinii pneumonia. Clin Nucl Med 1985; 10: 669-70.

11 Hoffer P B, Bekerman C, Henkin R E. Gallium-67 imaging. New York: John Wiley \& Sons, 1978.

12 Kramer E L, Sanger J J, Garay S M et al. Gallium-67 scans of the chest in patients with acquired immunodeficiency syndrome. J Nucl Med 1987; 28: 1107-14.

13 Krishnamurthy G T, Singhi V, Taylor R D, Ranganath K, Blahd W H. Radionuclide scanning in the evaluation of Kaposi sarcoma. Arch Intern Med 1976; 136: 400-3.

14 Levenson S M, Warren R D, Richman S D, Johnston G S, Chabner B A. Abnormal pulmonary gallium accumulation in $P$. carinii pneumonia. Radiology 1976; 119: 395-8.

15 Levin M, McLeod R, Young Q et al. Pneumocystis carinii pneumonia: importance of gallium scan for early diagnosis and description of a new immunoperoxidase technique to demonstrate Pneumocystis carinii. Am Rev Respir Dis 1983; 128: 182-5.

16 Malhotra C M, Erickson. A D, Feinsilver S $\mathrm{H}$, Spraragen S C, McCully K S, Claunch B C. Ga-67 studies in a patient with acquired immunodeficiency syndrome and disseminated mycobacterial infection. Clin Nucl Med 1985; 10: 96-8.

17 Picard C, Meignan M, Rosso J, Cinotti L, Mayaud C, Revuz J. Technetium-99m aerosol and gallium scanning in acquired immune deficiency syndrome. Clin Nucl Med 1987; 12: 501-6.

18 Skarzynski J J, Sherman W, Lee H K, Berger $\mathrm{H}$. Patchy uptake of gallium in the lungs of AIDS patients with atypical mycobacterial infection. Clin Nucl Med 1987; 12: 507-9.

19 Sollitto R A. Gallium scintigraphy useful in imaging AIDS infections. Diagn Imag Int 1987; 3/2: 38-41.
20) Stover D E, White D A, Romano P A Gellene R A, Robeson W A. Diagnosis of pulmonary disease in acquired immune deficiency syndrome (AIDS). Role of bronchoscopy and bronchoalveolar lavage. Am Rev Respir Dis 1984; 130: 639-62.

21 Stover D E, White D A, Romano P A, Gellene R A, Robeson W A. Spectrum of pulmonary diseases associated with the acquired immune deficiency syndrome. Am J Med 1985; 78: 429-37.

22 Szabados A, Schierz G, Deinhardt F. Die Pneumocystis carinii-Pneumonie. Internist 1985; 26: 335-40.

23 Tatsch K, Knesewitsch P, Küffer G, Dörner $G$, Göbel F D. The value of Ga-67 scintigraphy in patients with AIDS. Nuklearmedizin. Stuttgart - New York: F. K. Schattauer, 1988; 393-6.

24 Tuazon C U, Delaney M D, Simon G L, Witorsch P, Varma V M. Utility of gallium67 scintigraphy and bronchial washings in the diagnosis and treatment of Pneumocystis carinii pneumonia in patients with the acquired immune deficiency syndrome. Am Rev Respir Dis 1985; 132: 1087-92.

25 Turbiner E H, Yeh S D, Rosen P P, Bains M $S$, Benua R S. Abnormal gallium scintigraphy in Pneumocystis carinii pneumonia with a normal chest radiograph. Radiology 1978 127: 437-8.

26 Weiske R, Buck J, Schneider R, Hannemann Th, Krauss B. Lungenkomplikationen bei AIDS. Radiologe 1986; 26: 3-9.

27 Woolfenden J M, Carrasquillo J A, Larson S $M$ et al. Acquired immunodeficiency syndrome: Ga-67 citrate imaging. Radiology 1987; $162: 383-7$.

Korrespondenz:

Dr. med. K. Tatsch

Nuklearmedizinische Abteilung der Klinik und Poliklinik für Radiologie

Klinikum Großhadern

Marchioninistraße 15

D-8000 München 70, BRD 